\section{The SARS-CoV-2 pandemic: An urirgent need to relook at the training of the African health workforce}

To the Editor: As of 11 March 2020, the severe acute respiratory syndrome coronavirus 2 (SARS-CoV-2) pandemic had infected over 120000 people in more than 90 countries, of whom 4369 had died. ${ }^{[1]}$ As recent modelling analysis highlights, the evolving impact of SARS-CoV-2 will be as a large outbreak in sub-Saharan Africa (SSA) that may rapidly overwhelm existing health services. ${ }^{[2]}$ To avoid the worst of outcomes, African health systems must act quickly and decisively. Investing in health professions education will be essential to both containing and managing the pandemic and any other new infections in the future. Here we describe how a transnational HIV training project, implemented by the African Forum for Research and Education in Health (AFREhealth) ${ }^{[3]}$ and the University of California, San Francisco (UCSF), offers a template for how we can deploy African-bred approaches to address this emerging threat.

Starting in 2019, AFREhealth and UCSF embarked on a project called Strengthening InterProfessional Education to Improve HIV Care Across Africa (STRIPE HIV). STRIPE HIV aims to build health workforce capacity across Africa to achieve HIV epidemic control. It seeks to reshape how health professions training is delivered, emphasising the importance of team-based learning and continuous service quality improvement. With funding from the US President's Emergency Plan for AIDS Relief (PEPFAR), 20 health professions training institutions across SSA are using these training resources to equip early-career health professionals and undergraduate learners working in settings with the highest HIV burden. The project is now in its 10th month and its outcomes have been impressive. Snowballing enthusiasm and imaginative partnerships have enabled over 66 partner institutions to participate in training events across 14 countries. Furthermore, over 350 faculty and educators have facilitated training at over 100 clinical sites. To date, over 3500 learners from across medical, nursing, laboratory, pharmacy and other health professions training programmes have participated in training ( $\sim 65 \%$ undergraduate and $35 \%$ early career) in over 80 workshops.

STRIPE's progress to date offers two important insights. First, it illustrates the importance of the AFREhealth network as a powerful vehicle to enable rapid dissemination of evidence-based training for interprofessional HIV across Africa. AFREhealth provides an infrastructure for communities of practice that can support improvements in quality in undergraduate and early-career HIV training, as well as enhance health professions education and research. Secondly, the STRIPE programme offers a template for how interprofessional training can be leveraged to respond to the approaching coronavirus disease 2019 pandemic. While cases in Africa are still few, it is imperative that we equip frontline providers, from across the cadres, to respond appropriately. A cohesive, interprofessional response, informed by evidence-based guidelines and country guidelines, while continually addressing quality improvement, will be important in preventing ongoing transmission and offering compassionate care.

Time is short. Clear instruction on how to manage patients with coronavirus disease 2019 (COVID-19) must be delivered urgently to healthcare workers across Africa. AFREhealth's STRIPE project offers an illustration of how this can be done.

Funding. The project outlined was funded by PEPFAR via the Health Resources and Services Administration, USA.

\section{Michael Reid}

Department of Medicine, University of California, San Francisco, USA michael.reid2@ucsf.edu

\section{Fatima Suleman}

Pharmaceutical Sciences, College of Health Sciences, University of KwaZulu-Natal, Durban, South Africa

\section{Marietjie de Villiers}

Department of Family Medicine and Primary Care, Faculty of Medicine and Health Sciences, Stellenbosch University, Cape Town, South Africa

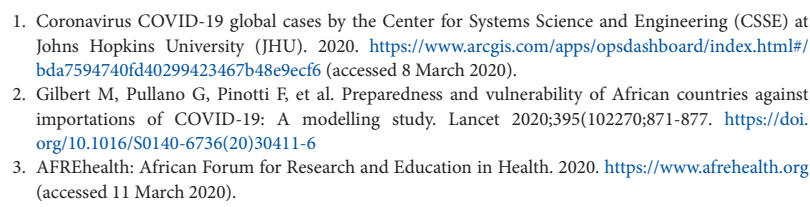

1. Coronavirus COVID-19 global cases by the Center for Systems Science and Engineering (CSSE) a Johns Hopkins University (JHU). 2020. https://www.arcgis.com/apps/opsdashboard/index.html\# bda7594740fd40299423467b48e9ecf6 (accessed 8 March 2020).

2. Gilbert M, Pullano G, Pinotti F, et al. Preparedness and vulnerability of African countries against importations of COVID-19: A modelling study. Lancet 2020;395(102270;871-877. https://doi. org/10.1016/S0140-6736(20)30411-6

3. AFREhealth: African Forum for Research and Education in Health. 2020. https://www.afrehealth.org (accessed 11 March 2020)

S Afr Med J 2020;110(4):261. https://doi.org/10.7196/SAMJ.2020.v110i4.14713 